\title{
130. On Pseudoparacompactness and Continuous Mappings
}

\author{
By Takao Hoshina \\ Tokyo University of Education \\ (Comm. by Kinjirô Kunugr, M. J. A., Sept. 12, 1972)
}

Throughout this paper we assume that spaces are completely regular $T_{1}$-spaces and maps are continuous. The completion of a space $X$ with respect to its finest uniformity is called the topological completion of $X$, and denoted by $\mu X$. According to Morita [8] a space $X$ is called pseudoparacompact (resp. pseudo-Lindelöf) if $\mu X$ is paracompact (resp. Lindelöf).

As for these notions, in the same paper Morita proved the following remarkable results.

Theorem 1 (Morita [8], Theorems 3.1, 3.2 and 3.5).

(1) $\mu X$ is compact iff $X$ is pseudocompact.

(2) $\mu X$ is always a paracompact $M$-space for any $M$-space $X$.

(3) Let $X$ be an $M$-space. $X$ is pseudo-Lindelöf iff it is the quasiperfect inverse image of a separable metric space.

The characterizations of pseudoparacompactness and pseudoLindelöfness have been obtained by Howes [4] and Ishii [5] independently. On the other hand, in [2] Hanai and Okuyama (cf. Isiwata [6]) essentially proved the following result: "If a space $X$ is the inverse image of a pseudocompact space under an open quasi-perfect map, then $X$ is pseudocompact". Here the assumption that the map is open cannot be dropped in general ([3] Example 2.4). Analogously to this result, in $\S 1$ we shall prove the following theorem which is a partial answer to a problem posed by Ishii [5] concerning (2) and (3) of Theorem 1: "Is pseudoparacompactness or pseudo-Lindelöfness preserved under taking the inverse image by a quasi-perfect (or perfect) map?"

Theorem 2. If there is an open quasi-perfect map $\varphi: X \rightarrow Y$ from a space $X$ onto a pseudoparacompact (resp. pseudo-Lindelöf) space $Y$, then $X$ is pseudoparacompact (resp. pseudo-Lindelöf).

In $\S 2$, by virtue of recent results obtained by Morita, we shall prove the following

Theorem 3. Let $\varphi: X \rightarrow Y$ be an open quasi-perfect map from a space $X$ onto a space $Y$.

(1) If $\mu Y$ is locally compact and paracompact, then so is $\mu X$.

(2) If $\mu Y$ is o-compact, then so is $\mu X$.

§1. Proof of Theorem 2. Before proving Theorem 2, we shall 
need some preliminalies. For a space $X$, let $\mu$ be the finest uniformity of $X$ and $\nu$ the uniformity of all countable normal coverings of $X$.

Lemma 1.1 (Howes [4]). A space $X$ is pseudoparacompact (resp. pseudo-Lindelöf) iff for any weakly Cauchy filter $\mathfrak{F}$ with respect to $\mu$ (resp. $\nu$ ) there exists a Cauchy filter (S) with respect to $\mu$ containing $\widetilde{F}$.

Here a filter $\mathfrak{f}$ in $X$ is called weakly Cauchy with respect to a uniformity $\mu$ of $X$ if for any uniform cover $\mathfrak{U}$ in $\mu$ there is a filter $\mathbb{S}$ in $X$ containing $\mathfrak{F}$ such that $G \subset U$ holds for some $G \in \mathbb{S}$ and $U \in \mathfrak{H}$.

Let $\mathcal{C}(X)$ be the family of all non-empty compact subsets of a given space $X$. Following the convention of [7], we topologize $\mathcal{C}(X)$ with the Vietoris topology; for a finite collection $\left\{U_{1}, U_{2}, \cdots, U_{n}\right\}$ of open sets, $\left\langle U_{1}, U_{2}, \cdots, U_{n}\right\rangle$ will denote the subset of $\mathcal{C}(X)$ to which the compact set $K$ belongs iff $K \subset \cup_{i} U_{i}$ and $K \cap U_{i} \neq \emptyset$ for $i=1,2, \cdots, n$. Open sets in $\mathcal{C}(X)$ are unions of an arbitrary number of these sets.

Lemma 1.2 (Michael [7]), $\mathcal{C}(X)$ is completely regular and $T_{1}$ iff $X$ is completely regular and $T_{1}$.

A space $X$ is called topologically complete if $\mu X=X$ (cf. [8]).

Lemma 1.3 (Zenor [10]). $\mathcal{C}(X)$ is topologically complete iff $X$ is topologically complete.

A subset $F$ of a space $X$ is called relatively pseudocompact if every real-valued continuous function over $X$ is bounded on $F$.

Lemma 1.4 (Dykes [1]). If $F$ is a relatively pseudocompact subset of a topologically complete space $X$, then $\mathrm{cl}_{X} F$ is compact.

A map $\varphi: X \rightarrow Y$ is called a $Z$-map if the image of each zero-set in $X$ is closed in $Y$. In [6], Isiwata extended the notion of $Z$-maps; a map $\varphi: X \rightarrow Y$ is a $W Z$-map if $\operatorname{cl}_{\beta X} \varphi^{-1}(y)=\beta(\varphi)^{-1}(y)$ for every $y$ in $Y$, where $\beta(\varphi)$ denotes the Stone extension of $\varphi$.

The following lemma is useful.

Lemma 1.5. Let $\varphi: X \rightarrow Y$ be a map from $X$ onto $Y$ such that $\varphi^{-1}(y)$ is relatively pseudocompact for each $y$ in $Y$. For $y$ in $Y$, let us put $\tilde{\varphi}(y)=\operatorname{cl}_{\mu X} \varphi^{-1}(y)$. If $\varphi$ is an open $W Z$-map, then the mapping $\tilde{\varphi}$ from $Y$ into $\mathcal{C}(\mu X)$ is continuous. Conversely if $\tilde{\varphi}$ is continuous then $\varphi$ is open, and moreover if $X$ is normal then $\varphi$ is closed.

Proof. Clearly $\tilde{\varphi}$ maps $Y$ into $\mathcal{C}(\mu X)$ by Lemma 1.4. Let $\varphi$ be an open $W Z$-map, and for $y$ in $Y$ let $\tilde{\varphi}(y) \in\left\langle U_{1}, U_{2}, \cdots, U_{n}\right\rangle$, where $U_{i}$ is an open set in $\mu X$ for each $i$. If we choose an open set $U^{\prime}$ in $\beta X$ such that $U^{\prime} \cap \mu X=\cup_{i} U_{i}$, then the set $V=\bigcap_{i} \varphi\left(U_{i} \cap X\right) \cap\left(\beta Y-\beta \varphi\left(\beta X-U^{\prime}\right)\right)$ is an open set in $Y$ containing $y$ since $\varphi$ is an open $W Z$-map and $\operatorname{cl}_{\mu X} \varphi^{-1}(y)$ is compact. Moreover we easily see $\tilde{\varphi}(V) \subset\left\langle U_{1}, U_{2}, \cdots, U_{n}\right\rangle$. Therefore $\tilde{\varphi}$ is continuous. Conversely let us assume $\tilde{\varphi}$ is continuous. Let $U$ be an open set in $X$ and choose an open set $U^{\prime}$ in $\mu X$ such that $U^{\prime} \cap X=U$. Then $\varphi(U)=\tilde{\varphi}^{-1}\left(\left\langle U^{\prime}, \mu X\right\rangle \cap \tilde{\varphi}(Y)\right)$. Hence $\varphi$ is open. Now, 
let us assume $X$ is normal. For a closed set $F$ in $X$, let us put $\widetilde{\mho}=\left\{K \in \mathcal{C}(\mu X) \mid \operatorname{cl}_{\mu X} F \cap K \neq \emptyset\right\}$. Then $\mathfrak{\mho}$ is closed in $\mathcal{C}(\mu X)$, and $\varphi(F)$ $=\tilde{\varphi}^{-1}(\mathfrak{\mho} \cap \tilde{\varphi}(Y))$. Therefore $\varphi$ is closed. This proves Lemma 1.5.

Theorem 2 is an immediate consequence of the following

Theorem 4. Let $\varphi: X \rightarrow Y$ be an open WZ-map from a space $X$ onto a pseudoparacompact (resp. pseudo-Lindelöf) space $Y$ such that $\varphi^{-1}(y)$ is relatively pseudocompact for each $y$ in $Y$, then $X$ is pseudoparacompact (resp. pseudo-Lindelöf).

Proof. Let $\mathfrak{\mho}$ be a weakly Cauchy filter in $X$ with respect to $\mu$ (resp. $\nu$ ). Then the filter $\varphi(\widetilde{F})$ is weakly Cauchy with respect to $\mu$ (resp. $\nu$ ) since $\varphi$ is continuous. Moreover since $Y$ is pseudoparacompact (resp. pseudo-Lindelöf), by Lemma 1.1 there exists a Cauchy filter (s) in $Y$ with respect to $\mu$, which contains $\varphi(\widetilde{F})$. Let $\tilde{\varphi}$ be a map as in Lemma 1.5 , then $\tilde{\varphi}(\mathbb{S})$ is also a Cauchy filter in $\mathcal{C}(\mu X)$ with respect to $\mu$ since $\tilde{\varphi}$ is continuous. Therefore since $\mathcal{C}(\mu X)$ is topologically complete by Lemma 1.3, $\tilde{\varphi}(\varangle)$ converges to some $K$ in $\mathcal{C}(\mu X)$. Let us suppose that $\cap\left\{\mathrm{cl}_{\mu X} F \mid F \in \mathscr{F}\right\} \cap K=\emptyset$. Since $K$ is compact, it follows that $\operatorname{cl}_{\mu X} F \cap K=\emptyset$ for some $F \in \mathfrak{\mho}$. This means that $K \in\left\langle\mu X-\operatorname{cl}_{\mu X} F\right\rangle$. Since $\tilde{\varphi}(\mathbb{S})$ converges to $K$, there exists $G$ in $\mathbb{S}$ such that $\tilde{\varphi}(G) \subset\left\langle\mu X-\operatorname{cl}_{\mu X} F\right\rangle$. Then it is easily seen that $\varphi^{-1} G \subset X-F$. But this contradicts that $\varphi(\mathfrak{F}) \subset \mathbb{S}$. Hence $\mathfrak{F}$ has a cluster point in $K$. This shows that $\mathfrak{F}$ is contained in a Cauchy filter in $X$ with respect to $\mu$. Therefore $X$ is pseudoparacompact (resp. pseudo-Lindelöf) by Lemma 1.1. The proof is completed.

Remark. Under the map $\varphi: X \rightarrow Y$ given in Theorem 4, let us assume that $Y$ is pseudocompact and consider $\mathfrak{F}$ in the proof above to be a weakly Cauchy filter with respect to the uniformity of all finite normal coverings, then under the same argument as above, by ([4], Theorem 3) we can conclude that $X$ is pseudocompact. This is an another proof of ([6], Theorem 4.2).

As an application of Theorem 4 we have

Theorem 5. Let $X$ be a pseudocompact space and $Y$ a first countable and pseudoparacompact (resp. pseudo-Lindelöf) space. Then $X \times Y$ is pseudoparacompact (resp. pseudo-Lindelöf).

Proof. Since the projection $X \times Y \rightarrow Y$ is a $Z$-map by ([6], Theorem 2.1), this follows from Theorem 4.

§ 2. Proof of Theorem 3. Theorem 3 is a direct consequence of the following lemma and theorems which are due to Morita.

Lemma 2.1. Let $\varphi: X \rightarrow Y$ be an open WZ-map from $X$ onto $Y$ such that $\varphi^{-1}(y)$ is relatively pseudocompact for each $y$ in $Y$. If $F$ is a relatively pseudocompact subset of $Y$, then $\varphi^{-1}(F)$ is relatively pseudocompact. 
Proof. For any real-valued continuous function $f$ on $X$, let us define real-valued functions $f^{s}$ and $f^{i}$ on $Y$ by

$$
f^{s}(y)=\sup \left\{f(x) \mid x \in \varphi^{-1}(y)\right\}, \quad f^{i}(y)=\inf \left\{f(x) \mid x \in \varphi^{-1}(y)\right\} .
$$

Then $f^{s}$ and $f^{i}$ are continuous by ([6], Lemma 4.1) and bounded on $F$. Hence $f$ is bounded on $\varphi^{-1}(F)$ and this proves Lemma 2.1.

Theorem 6 (Morita [9]). For a space $X, \mu X$ is locally compact and paracompact iff there exists a normal open covering of $X$ consisting of relatively pseudocompact subsets.

Theorem 7 (Morita). For a space $X, \mu X$ is o-compact iff $X$ is expressed as a union of a countable number of relatively pseudocompact subsets.

Proof. Let $\mu X=\cup\left\{K_{i} \mid i=1,2, \cdots\right\}$, where each $K_{i}$ is compact. Then $X=\cup_{i}\left(K_{i} \cap X\right)$ and since $X$ is $C$-embedded in $X$ by ([8], Theorem 2.4), $K_{i} \cap X$ is relatively pseudocompact. Conversely, suppose that $X=\cup\left\{F_{i} \mid i=1,2, \cdots\right\}$, where each $F_{i}$ is relatively pseudocompact. Let us put $Y=\bigcup_{i} \mathrm{cl}_{\mu X} F_{i}$. Then $X \subset Y \subset \mu X$ and $Y$ is a $\sigma$-compact space by Lemma 1.4. Therefore by ([8], Theorem 2.5) it holds that $Y=\mu X$. Hence $\mu X$ is $\sigma$-compact and this completes the proof of Theorem 7 .

\section{References}

[ 1 ] N. Dykes: Mappings and realcompact spaces. Pacific J. Math., 31, 347357 (1969).

[2] S. Hanai and A. Okuyama: On pseudocompactness and continuous mappings. Proc. Japan Acad., 38, 444-447 (1962).

[3] M. Henriksen and J. R. Isbell: Some properties of compactifications. Duke Math. J., 25, 83-105 (1958).

[ 4] N. R. Howes: On completeness. Pacific J. Math., 38, 431-440 (1971).

[5] T. Ishii: Some theorems related to pseudoparacompactness (to appear).

[6] T. Isiwata: Mappings and spaces. Pacific J. Math., 20, 455-480 (1967).

[ 7 ] E. Michael: Topologies on spaces of subsets. Trans. Amer. Math. Soc., 71, 151-182 (1951).

[ 8 ] K. Morita: Topological completions and $M$-spaces. Sci. Rep. Tokyo Kyoiku Daigaku, 10, 271-288 (1970).

[9] _ - On the dimension of the product of Tychonoff spaces (to appear).

[10] P. Zenor: On the completeness of the space of compact subsets. Proc. Amer. Math. Soc., 24, 190-192 (1970). 